\title{
Excess medical costs in patients with asthma and the role of comorbidity
}

\author{
Wenjia Chen ${ }^{1,2}$, Larry D. Lynd ${ }^{1,3}$, J. Mark FitzGerald ${ }^{2,4}$, Carlo A. Marra ${ }^{5}$, \\ Robert Balshaw ${ }^{6}$, Teresa To $^{7}$, Hamid Tavakoli ${ }^{2,4}$ and Mohsen Sadatsafavi ${ }^{1,2,4}$ for \\ the Canadian Respiratory Research Network
}

\begin{abstract}
Affiliations: ${ }^{1}$ Collaboration for Outcomes Research and Evaluation, Faculty of Pharmaceutical Sciences, University of British Columbia, Vancouver, BC, Canada. ${ }^{2}$ Institute for Heart and Lung Health, Division of Respiratory Medicine, Faculty of Medicine, University of British Columbia, Vancouver, BC, Canada. ${ }^{3}$ Centre for Health Evaluation and Outcome Sciences, Providence Health, St. Paul's Hospital, Vancouver, BC, Canada. ${ }^{4}$ Centre for Clinical Epidemiology and Evaluation, University of British Columbia, Vancouver, BC, Canada. ${ }^{5} \mathrm{New}$ Zealand's National School of Pharmacy, University of Otago, Dunedin, New Zealand. ${ }^{6} \mathrm{BC}$ Centre for Disease Control, Vancouver, BC, Canada. ${ }^{7}$ The Hospital for Sick Children, Toronto, ON, Canada.
\end{abstract}

Correspondence: Wenjia Chen, Collaboration for Outcomes Research and Evaluation, 2405 Wesbrook Mall, Vancouver, BC, Canada, V6T 1Z3. E-mail: wenjia.chendalumni.ubc.ca

ABSTRACT Asthmatic patients frequently have comorbidities, but the role of comorbidities in the economic burden of asthma is unclear. We examined the excess direct medical costs, including asthmaand comorbidity-related costs, in patients with asthma.

We created a propensity score-matched cohort of patients newly diagnosed with asthma and nonasthmatic comparison subjects, both aged 5-55 years, from health administrative data (1997-2012) in British Columbia, Canada. Health services use records were categorised into 16 major disease categories based on International Classification of Diseases codes. Excess costs (in 2013 Canadian dollars (\$)) were estimated as the adjusted difference in direct medical costs between the two groups.

Average overall excess costs were estimated at \$1058/person-year (95\% CI 1006-1110), of which \$134 (95\% CI 132-136) was attributable to asthma and \$689 (95\% CI 649-730) to major comorbidity classes. Psychiatric disorders were the largest component of excess comorbidity costs, followed by digestive disorders, diseases of the nervous system, and respiratory diseases other than asthma. Comorbidityattributable excess costs greatly increased with age but did not increase over the time course of asthma.

These findings suggest that both asthma and comorbidity-related outcomes should be considered in formulating evidence-based policies and guidelines for asthma management.

@ERSpublications

Costs of comorbidities are much higher than costs associated with asthma itself http://ow.ly/Lutb3031Pye

This article has supplementary material available from erj.ersjournals.com

Received: June 082016 | Accepted after revision: Aug 102016 | First published online: Oct 062016

Support statement: This study was funded by the Canadian Respiratory Research Network (CRRN). CRRN is supported by grants from the Canadian Institutes of Health Research (CIHR) Institute of Circulatory and Respiratory Health, Canadian Lung Association (CLA)/Canadian Thoracic Society (CTS), British Columbia Lung Association, and industry partners Boehringer-Ingelheim Canada Ltd, AstraZeneca Canada Inc., and Novartis Canada Ltd. Funding for training of graduate students and new investigators within the network was supported by the above funding sponsors, as well by GlaxoSmithKline Inc. The funders had no role in the study design, data collection and analysis, or preparation of the manuscript. M. Sadatsafavi receives salary support from the Canadian Institutes of Health Research and the Michael Smith Foundation for Health Research. Funding for this article has been deposited with the Open Funder Registry

Conflict of interest: None declared.

Copyright OERS 2016 


\section{Introduction}

Asthma is a prevalent chronic disease that affects roughly 300 million people worldwide [1]. Approximately $8.6 \%$ of the world's young adults and $14.0 \%$ of children experience asthma symptoms [1]. The substantial economic burden of asthma has been documented in many large-scale cross-sectional epidemiological studies [2, 3], including those conducted in the USA [4], Europe [5] and Canada [6].

Individuals with asthma are at greater risk of multimorbidity compared to the general population $[7,8]$. The presence of comorbidities in asthma is associated with suboptimal asthma control [9], increased healthcare resource utilisations [10], lower quality of life [11] and reduced efficacy of asthma treatments [12]. For this reason, both asthma and comorbidity-related outcomes should be considered in formulating evidence-based policies and guidelines for asthma management. This requires valid and current evidence about the extent of comorbidity burden in people with asthma. Failure to account for the economic impact of comorbidities in asthma leads to not only biased estimation of the true burden of asthma but also underestimation of the cost-effectiveness of interventions that aim to prevent or manage comorbidities or improve the overall health of asthmatic patients. Understanding the extent of the comorbidity burden can further generate hypotheses regarding the systemic aspects of asthma.

Our primary objective was to estimate excess direct medical costs (hereafter referred to as "excess costs" for brevity) in individuals with asthma. The secondary objectives were to compare the share of asthma and major comorbid categories in excess costs, and to evaluate variations in excess costs across age groups and over the time course of asthma.

\section{Materials and methods}

\section{Data sources}

We retrieved data from January 1997 to December 2012 from the provincial health administrative databases of British Columbia (BC), a large province in Canada with 4.4 million residents as of 2011 [13]. These databases provide linked, individual-level information on demographics, vital statistics and healthcare encounters of all BC residents [14-18] (supplementary material S1). All inferences, opinions and conclusions drawn in this study are those of the authors, and do not reflect the opinions or policies of the Data Steward(s). Ethical approval was obtained from the University of British Columbia Human Ethics Board (H08-01287).

\section{Study design and sample}

This was a retrospective, propensity score-matched cohort study. The asthma cohort consisted of individuals newly identified with asthma. We first identified all individuals who satisfied a validated case definition of asthma [19], defined as the presence of, during any rolling 12-month period, one or more asthma-specific inpatient encounters (with the most responsible diagnosis being asthma), two or more asthma-related outpatient visits (in different dates), or three or more filled asthma-related prescriptions. Asthma-specific inpatient and outpatient encounters were determined based on International Classification of Diseases (ICD) codes (ICD-9: 493.x; ICD10: J45.x, J46.x). Asthma-related medications were pre-specified (supplementary material S2). The index date was defined as the date of the patient's first asthma-related inpatient or outpatient service based on their history of resource use, marking the beginning of follow-up. The cohort included individuals aged between 5 and 55 years at their index dates. To include only new patients, we restricted the sample to individuals who were registered with the healthcare system for at least 300 days per year (i.e. fully registered) for at least 5 years prior to the index date without having any asthma-related records. These individuals also had to have at least 1 year of follow-up data after the index date.

Because asthma medications significantly overlap with treatment for chronic obstructive pulmonary disease (COPD), to minimise the chance of including patients with COPD who received the same drugs, we excluded patients with any COPD-specific healthcare resource usage within 2 years of the asthma index date (COPD codes: ICD-9: 491.x, 492.x, 493.2x, 496.x; ICD-10: J43.x, J44.x). Patients with asthma can develop COPD [20], which is a legitimate comorbidity to consider in this study. Therefore, COPD-related records beyond the first 2 years after the asthma index date were included.

\section{Propensity score matching}

As the basis of our comparator group, we had access to a random sample of $100000 \mathrm{BC}$ residents who had no asthma-specific health resource use between January 1997 and December 2012. From this sample we created a comparison group. The BC Ministry of Health does not permit researchers to access the full provincial population due to privacy considerations.

We used propensity score matching to create a balanced 1:1 cohort of asthmatic and non-asthmatic subjects, as follows: First, we excluded non-asthmatic subjects with less than 5 years of coverage in the database, and defined the first day following the five continuous years of coverage for each remaining individual as their index date (to match the 5-year pre-index criterion applied to the asthma cohort). Comparison subjects also 
had to be between 5 and 55 years of age on their index date. We then merged the asthma and comparison cohorts, and fitted a logistic regression model predicting the probability that an individual had asthma. This model included age, sex, neighbourhood household income quintile, health services delivery area (all as observed in the index year) and baseline comorbidity status (observed in the 12 months before the index date). The latter was measured by the number of non-asthma-related hospitalisations, physician visits and medication dispensations, as well as a Charlson comorbidity index (CCI, excluding asthma from the score) [21]. Finally, we used the estimated propensity scores to perform matching based on nearest distance criterion, so that each member of the asthma cohort was matched to a member of the comparison cohort. Because the size of the pre-matching asthma cohort was larger than the comparison cohort (145744 versus 50110 , see supplementary material S3), we adopted the matching-with-replacement strategy (in which an individual in the comparison cohort could be matched to more than one patient in the asthma cohort). This approach allowed us to efficiently use the comparison cohort subject and has proven to yield results comparable to the conventional matching-without-replacement method [22].

All study subjects were followed from the index date to date of death, loss to follow-up (i.e. end of registration with the healthcare system), or December 31, 2012, whichever came first. Supplementary material S4 provides a schematic presentation of the study design.

\section{Outcomes}

Our primary outcome was all-cause excess costs, defined as the difference in all-cause direct medical costs between the asthma and comparison cohorts. Costs were summed from three components, comprising inpatient encounters, outpatient encounters and filled prescriptions, and were converted to 2013 Canadian dollars (\$) using the historical inflation rates [23]. Inpatient costs were calculated using the case mix methodology by multiplying the resource intensity weight (available for each hospitalisation record) with the average costs of hospitalisation in BC for the same fiscal year [24]. Outpatient and medication costs were directly available in the data. Costs of emergency department services were mostly captured by fee-for-service payments to physicians, with those leading to an inpatient episode captured by the corresponding inpatient records [25].

The secondary outcomes were condition-attributable excess costs, which were calculated by attributing resource use records to asthma, or to any of the 16 major comorbid areas as defined by the major disease categories in the ICD-10 system [26]. Resource uses attributable to the presence of symptoms categorised by the ICD-10 code range R00-R69 were assigned to related comorbid categories if the diagnosis code was grouped into that category in the Diagnosis-Related Groups based on General Equivalence Mappings [27]. ICD-9 codes, being used in the outpatient databases and up to 2002 in the inpatient databases, were converted to ICD-10 codes using validated cross-walk tables [28]. For medication records, we mapped the American Hospital Formulary Service (AHFS) major categories (available in the data) to ICD-10 categories [29] (supplementary material S5). Some records of resource use and medication dispensations could not be assigned to any disease or symptom categories (e.g. codes for injury, poisoning, burns and other external causes, laboratory tests, miscellaneous drugs, or when there were no ICD or AHFS codes). We grouped costs associated with such records into an "unattributable" category.

\section{Statistical analysis}

Descriptive analyses were performed using SAS 9.3 (SAS Institute Inc., Cary, NC, USA). Regression analyses were performed using Stata/IC (V.12.1. College Station, TX, USA). Differences in the distribution of matching variables between asthma and comparison cohorts were compared using the standardised difference, with a value below 0.20 indicating similarity between two groups [30].

The unit of analysis was person-year (PY). Follow-up time was divided into 12-month intervals starting from the index date, with the last interval potentially truncated. To avoid under-reporting due to individuals with temporary absences from the province, intervals with less than 300 days were removed unless death occurred. We used a multivariate general linear model with interval-specific costs as the dependent variable. Normal distribution and identity link were used, in line with expert recommendations on their robustness with large sample sizes [31]. Separate regression analyses were performed for the overall costs and by cost components. For all models, the independent variables included disease status (asthma $=1$, non-asthma=0), age group at the beginning of the interval (5-18 years, $19-45$ years, $46-55$ years), and the number of intervals since the index date. The models also included calendar year at the beginning of each interval.

Excess costs were estimated as the adjusted differences in the predicted costs between an asthmatic patient and the non-asthmatic match. Changes over age and time were captured by the regression coefficients for the three-way interaction between asthma status, age group and time from the index date. Because individuals in the comparison cohort could be matched to several individuals in the asthma cohort, our data were clustered around each comparator and all potential matches. Accordingly, we fitted a weighted regression as 
recommended [22], with comparison subjects weighted by the inverse of the frequency that they were matched to an asthmatic patient. We followed expert recommendation to use generalised estimating equations with the robust variance estimator to obtain valid inference for the nested clustered data [32].

\section{Sensitivity analysis}

To evaluate the robustness of our results against design features, we performed several sensitivity analyses. First, we repeated the cost analyses among individuals with short follow-up time $(<12$ months) who were excluded from the main analysis, adjusting for the length of follow-up in days. Second, to further minimise the risk of including patients with COPD, we estimated costs by removing all patient-years in which patients were over 45 years of age.

\section{Results}

We matched 134941 asthmatic patients to 31372 unique non-asthmatic subjects in the comparison group, with some comparator subjects selected to match more than one asthmatic patient (supplementary material S3). Table 1 compares the baseline characteristics of the two cohorts. Standardised differences were all below 0.10 , indicating appropriate matching [30].

\section{Prevalence and excess costs, overall and by conditions}

Table 2 shows that patients with asthma more frequently experienced comorbidities compared to those in the non-asthma cohort during the follow-up period (99\% versus 96\%), especially for respiratory diseases other than asthma (85\% versus 63\%), as well as infectious and parasitic diseases ( $48 \%$ versus $43 \%)$. Meanwhile, asthma cohorts experienced lower rates of pregnancy, childbirth and puerperium ( $8 \%$ versus $11 \%$ ).

Table 3 shows the adjusted excess costs across cost components and age groups. All-cause excess costs were $\$ 1058$ per PY (95\% CI 1006-1110) in asthmatic patients. Among these, $13 \%$ were attributable to asthma ( $\$ 134,95 \%$ CI $132-136)$, and 65\% were attributable to comorbidities ( $\$ 689,95 \%$ CI $649-730)$. The remaining $22 \%$ were unattributable to any disease and were mainly driven by outpatient services without recorded diagnoses or with diagnoses of general symptoms, laboratory tests and external causes. This component was excluded from further consideration.

Figure 1 shows that significant incremental costs were found for all included comorbidity categories in asthmatic patients. Excess costs were the highest for psychiatric disorders (\$167 per PY, 95\% CI 149-184]), followed by digestive disorders (\$79 per PY, 95\% CI 70-89), diseases of the nervous system (\$73 per PY, 95\% CI 66-81) and

TABLE 1 Baseline characteristics of the asthma cohort and the non-asthma comparison group

\begin{tabular}{|c|c|c|c|}
\hline & \multicolumn{2}{|c|}{ Study cohort } & \multirow{2}{*}{$\begin{array}{l}\text { Standardised } \\
\text { difference }\end{array}$} \\
\hline & Asthma & Non-asthma\# & \\
\hline Subjects & 134941 & 134941 & \\
\hline Age years & $27.7 \pm 15.8$ & $27.6 \pm 15.6$ & 0.01 \\
\hline Age group & & & 0.07 \\
\hline $5-18$ years & $37.0 \%$ & $34.4 \%$ & \\
\hline 19-45 years & $45.4 \%$ & $49.1 \%$ & \\
\hline$>45$ years & $17.5 \%$ & $16.5 \%$ & \\
\hline Female & $56.1 \%$ & $56.7 \%$ & 0.01 \\
\hline $\mathrm{CCl}^{+} \S$ & $0.2 \pm 0.6$ & $0.2 \pm 0.8$ & 0.00 \\
\hline Non-asthma inpatient visits ${ }^{\S}$ & $0.1 \pm 0.5$ & $0.1 \pm 0.5$ & -0.01 \\
\hline Non-asthma outpatient visits ${ }^{\S}$ & $10.4 \pm 12.8$ & $10.8 \pm 14.1$ & -0.03 \\
\hline Non-asthma prescriptions ${ }^{\S}$ & $9.6 \pm 44.8$ & $8.3 \pm 42.2$ & 0.03 \\
\hline Neighbourhood household income quintiles & & & 0.05 \\
\hline Q1: lowest quintile (lowest 20\%) & $20.2 \%$ & $18.9 \%$ & \\
\hline Q2: second quintile (20-40\%) & $20.5 \%$ & $19.8 \%$ & \\
\hline Q3: middle quintile (40-60\%) & $19.9 \%$ & $20.1 \%$ & \\
\hline Q4: fourth quintile $(60-80 \%)$ & $19.4 \%$ & $20.5 \%$ & \\
\hline Q5: top quintile $(80-100 \%)$ & $18.0 \%$ & $19.0 \%$ & \\
\hline Missing & $2.0 \%$ & $1.7 \%$ & \\
\hline
\end{tabular}

Data are presented as mean \pm SD, unless otherwise stated. $C C I$ : Charlson comorbidity index; Q: quintile. \#: from 31372 unique individuals selected with replacement for matching. ": difference in means or proportions divided by standard error. Imbalance was defined as absolute value $>0.10 .^{+}$: excluding asthma from the score. §: measured in the past 12 months before the index date. 
TABLE 2 Prevalence of condition-related healthcare resource use during the follow-up period

\begin{tabular}{|c|c|c|c|}
\hline & \multicolumn{2}{|c|}{ Study cohort } & \multirow{2}{*}{$\begin{array}{l}\text { Standardised } \\
\text { difference }\end{array}$} \\
\hline & Asthma & Non-asthma" & \\
\hline Subjects & 134941 & 134941 & \\
\hline Overall & $98.9 \%$ & $95.8 \%$ & 0.19 \\
\hline Respiratory diseases other than asthma & $84.9 \%$ & $63.4 \%$ & 0.51 \\
\hline Infectious and parasitic diseases & $48.3 \%$ & $42.7 \%$ & 0.11 \\
\hline Digestive disorders & $43.4 \%$ & $39.5 \%$ & 0.08 \\
\hline Eye, ear, nose & $66.9 \%$ & $64.2 \%$ & 0.06 \\
\hline Diseases of musculoskeletal and connective tissue & $61.0 \%$ & $58.1 \%$ & 0.06 \\
\hline Psychiatric disorders & $46.9 \%$ & $44.0 \%$ & 0.06 \\
\hline Diseases of nervous system & $47.5 \%$ & $45.3 \%$ & 0.04 \\
\hline Diseases of circulatory system & $31.6 \%$ & $30.3 \%$ & 0.03 \\
\hline Complications related to congenital abnormalities & $4.2 \%$ & $3.6 \%$ & 0.03 \\
\hline Hematologic disorders & $12.7 \%$ & $11.9 \%$ & 0.02 \\
\hline Perinatal-originated conditions & $2.1 \%$ & $1.9 \%$ & 0.01 \\
\hline Diseases of skin and subcutaneous tissue & $69.1 \%$ & $69.6 \%$ & -0.01 \\
\hline Endocrine, nutritional and metabolic diseases & $67.5 \%$ & $68.4 \%$ & -0.02 \\
\hline Genitourinary diseases & $55.2 \%$ & $56.9 \%$ & -0.03 \\
\hline Neoplasms & $22.9 \%$ & $26.3 \%$ & -0.08 \\
\hline Pregnancy, childbirth and puerperium & $7.7 \%$ & $10.5 \%$ & -0.10 \\
\hline
\end{tabular}

\#: from 31372 unique individuals selected with replacement for matching. ": difference in means or proportions divided by standard error. Imbalance was defined as absolute value $>0.10$.

respiratory diseases other than asthma (\$71 per PY, 95\% CI 66-76) (figure 1). Medication costs were the major driver in excess costs attributable to psychiatric disorders, digestive disorders and nervous diseases (49\%, 51\% and $82 \%$, respectively). Hospitalisation costs were prominent in psychiatric disorders, digestive disorders and respiratory diseases other than asthma ( $27 \%, 33 \%$ and $48 \%$ of excess costs, respectively).

Excess costs across age groups

Figure 2 shows excess costs across age groups. Among individuals aged 5-18 years, excess costs were $\$ 387$ per PY (95\% CI 341-433), with \$108 (28\%) attributable to asthma and \$200 (52\%) to comorbidities. The corresponding estimates for individuals aged 19-45 years were \$1103 (95\% CI 1042-1166), with \$121 (11\%) attributable to asthma and $\$ 735$ (67\%) to comorbidities; for individuals aged $>45$ years, excess costs were $\$ 1721$ per PY (95\% CI 1613.7-1828.9), with $\$ 184$ (11\%) attributable to asthma and $\$ 1149$ (67\%) to comorbidities. The greatest increases over age were found in costs of psychiatric disorders (from $\$ 36$ per PY for 5-18 years of age to $\$ 232$ per PY for $>45$ years of age) and diseases of the circulatory system (from $\$ 0$ per PY to $\$ 152$ per PY).

TABLE 3 Adjusted excess costs of asthma per person-year during the follow-up period

\section{Mean excess costs of asthma} versus non-asthma $(95 \%$ Cl) CAD\$

\begin{tabular}{|c|c|c|c|}
\hline All-cause costs & Asthma-attributable costs & $\begin{array}{l}\text { Costs attributable to } \\
\text { major comorbidity categories }\end{array}$ & Unattributable costs ๆ \\
\hline $1058.2(1006.1-1110.2)$ & $133.6(131.6-135.7)$ & $689.3(648.6-730.0)$ & $235.2(219.7-250.8)$ \\
\hline $259.7(235.1-284.2)$ & $5.6(5.0-6.3)$ & $217.5(196.4-238.6)$ & $36.6(28.1-45.0)$ \\
\hline $353.5(343.0-364.0)$ & $29.7(29.5-30.0)$ & $188.9(182.0-195.7)$ & $134.9(130.6-139.2)$ \\
\hline $445.0(419.5-470.5)$ & $98.3(96.5-100.1)$ & $282.9(263.6-302.3)$ & $63.8(54.7-72.8)$ \\
\hline 386.8 (340.9-432.8) & $108.1(106.0-110.1)$ & $200.2(163.9-236.4)$ & $78.6(61.7-95.5)$ \\
\hline $1103.1(1040.5-1165.6)$ & $121.3(118.8-123.8)$ & $735.2(686.3-784.0)$ & $246.6(225.1-268.1)$ \\
\hline $1721.3(1613.7-1828.9)$ & $184.2(179.2-189.2)$ & $1148.8(1063.2-1234.4)$ & $388.2(357.5-419.0)$ \\
\hline
\end{tabular}

\# : the major comorbidity categories were defined by the International Classification of Diseases (ICD)-10 major disease categories and related symptoms. ": included general symptoms, signs and findings; injury; poisoning; burning and external causes; factors influencing health status; other special purposes; the American Hospital Formulary Service (AHFS) miscellaneous drug group; no ICD code; and no AHFS code. 


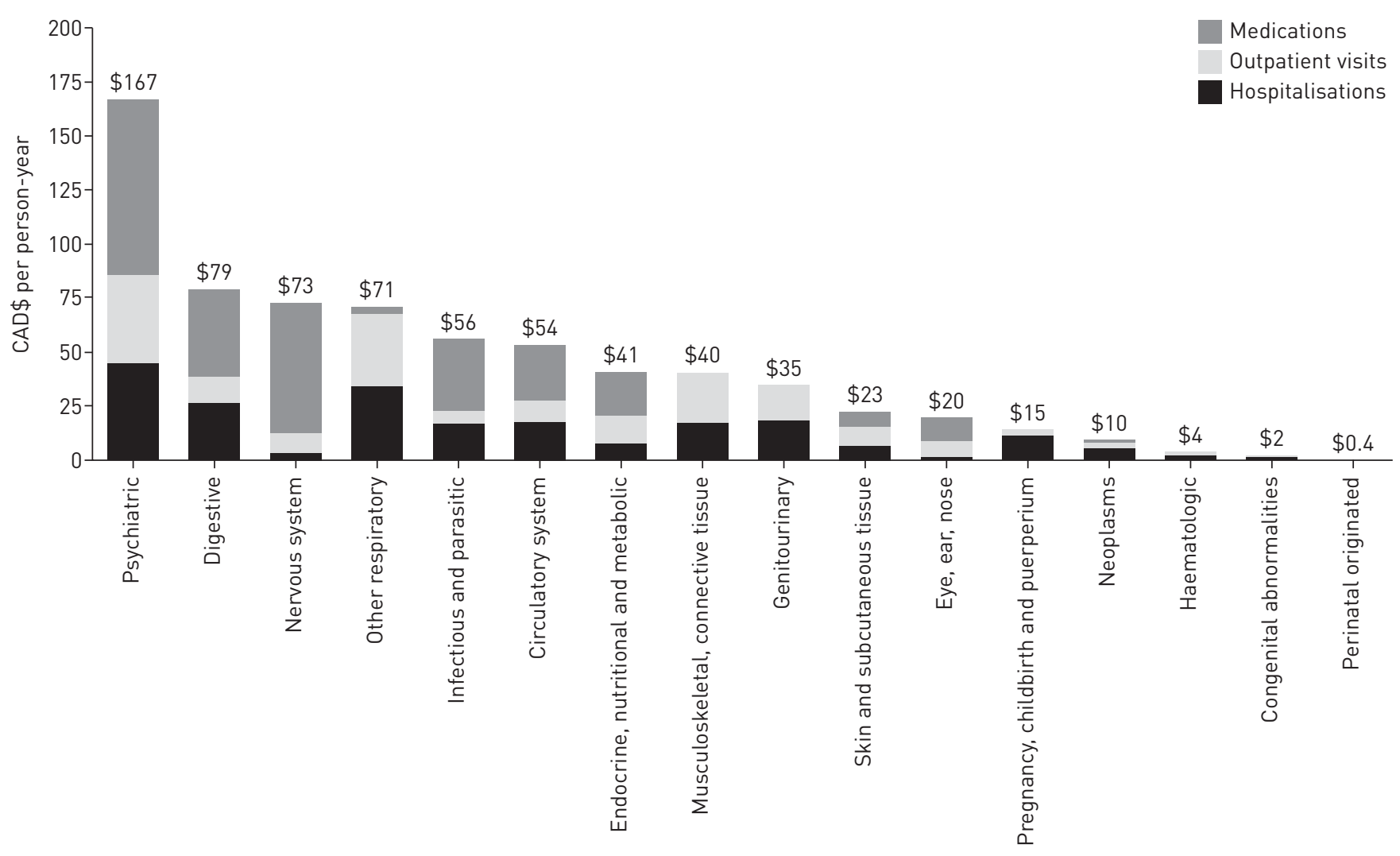

FIGURE 1 Adjusted annual excess costs by attribution to asthma and comorbidities, by cost components.

\section{Trend of excess costs over the time course of asthma}

Figure 3 shows the trend of excess costs within each age group over the 10 years from the diagnosis of asthma. Asthma-attributable excess costs consistently decreased over time from the index date across all age groups, with greater decreases found in younger individuals. For age groups of 5-18 years and 19-45 years, comorbidity-attributable excess costs decreased over time (5-18 years: $-\$ 26$ per PY per year, 95\% CI -39 to -13 ; $19-45$ years: $-\$ 22$ per PY per year, $95 \%$ CI -31 to -13 ). For those aged $>45$ years, comorbidity-attributable excess costs did not significantly change ( $\$ 5$ per PY, 95\% CI -11 to 22 ).

\section{Sensitivity analyses}

In the sensitivity analyses, asthmatic patients with $<12$ months of follow-up incurred $\$ 1607$ per PY (95\% CI 1356-1859) excess costs, which was only minimally different than the first-year costs in the main sample. When we removed person-years where subjects were $>45$ years of age, overall excess costs dropped by roughly $\$ 200$ (to $\$ 820$ per PY, 95\% CI 775-864) but the proportions of asthma-attributable and comorbidity-attributable costs stayed almost the same as in the main analysis (\$115 per PY (14\%) and \$521 per PY (64\%), respectively).

\section{Discussion}

Previous studies have shown that asthma is associated with increased prevalence of comorbidities $[7,8]$. Our study is the first to comprehensively examine the direct medical costs in patients with incident asthma by accounting for both asthma and asthma-related comorbidities. By estimating the excess costs incurred by asthmatic patients over and beyond the costs of matched non-asthmatic individuals, we found comorbidity-attributable costs (\$689 per PY) to be five-times higher than asthma-attributable costs ( $\$ 134$ per PY) in patients with asthma. This is despite the fact that the comorbidity profiles of the asthma group and the comparison group were similar before the start of follow-up. Psychiatric disorders were the largest component of comorbidity costs, followed by digestive diseases, diseases of the nervous system and respiratory diseases other than asthma. Although comorbidity-attributable excess costs greatly increased with age, they did not increase over the course of asthma. Our estimate of asthma-attributable costs aligned well with previous estimates $[6,33]$. The estimate of excess costs in asthmatic children was consistent with a study performed in the USA [34]. 


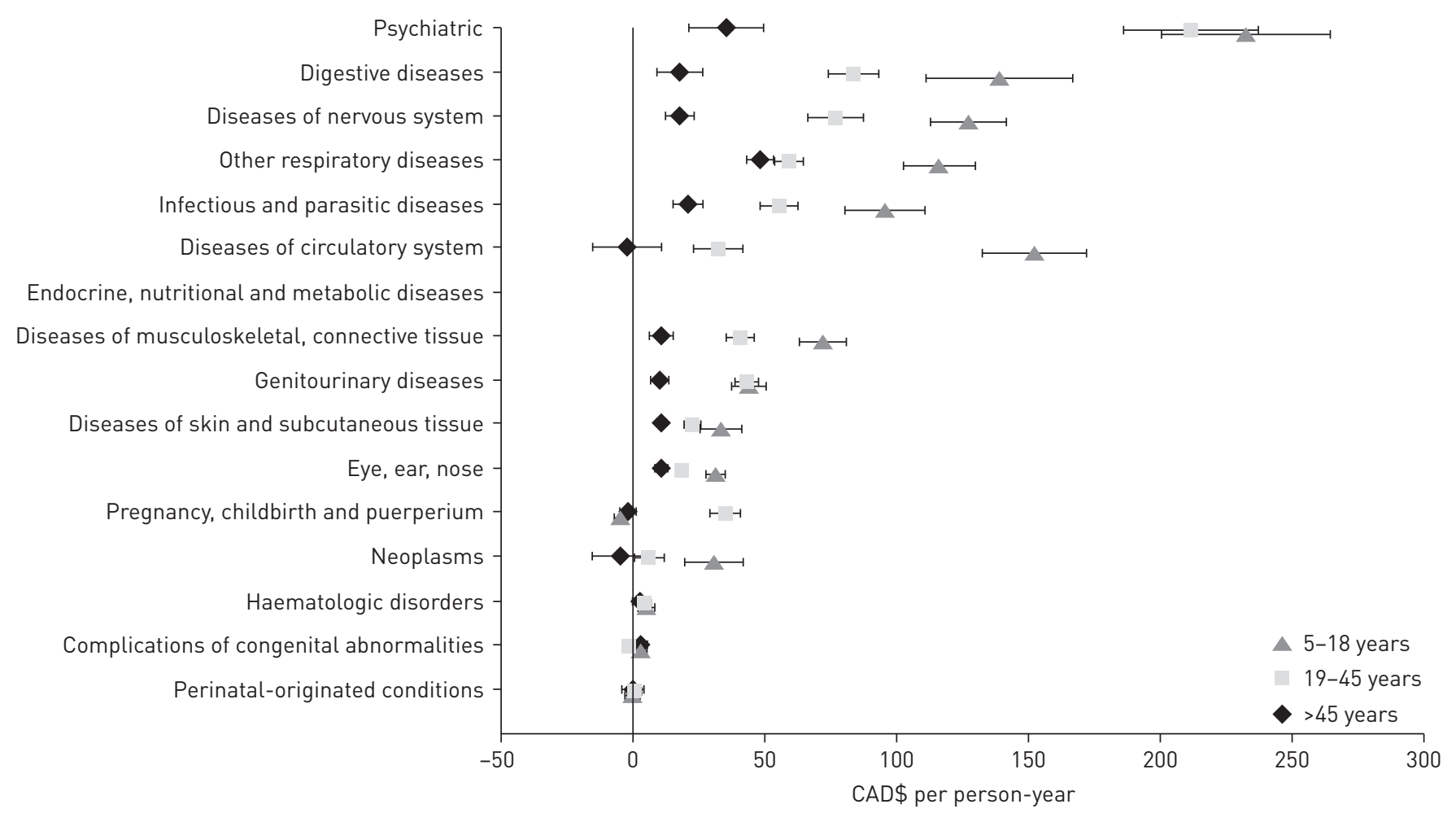

FIGURE 2 Adjusted annual excess costs by attribution to asthma and comorbidities, by age groups.

Findings from this study can be hypothesis-generating with regards to the relationship between asthma and comorbidity. Common inflammatory mechanisms between certain comorbidities and asthma could be responsible for their observed high burden [20]; COPD is one such example. We purposefully excluded patients with COPD-related resource use around the index date to ensure that patients with COPD, who have similar drug use patterns to asthmatic patients at early stages, were not erroneously included in the cohort. However, the subsequent high costs of respiratory diseases other than asthma suggest that some remaining asthmatic individuals still developed COPD over time, which is highly costly in itself and also a risk factor for other conditions such as cardiovascular diseases [35]. In addition, asthma activity or its treatment can affect the incidence or intensity of comorbid disease. For instance, the burden of psychological disorders could be due to asthma impairment and suboptimal asthma control [36], but corticosteroid use is also associated with the development of such comorbidities [37]. Comorbid conditions can also complicate treatment strategies, increasing the need for medications and the risk of adverse events, which eventually increases the overall excess costs. Lastly, while it was not surprising that comorbidity-attributable excess costs increased with age, they decreased over the time course of asthma in younger adults while staying constant in older adults. It is possible that acute onset of asthma boosted costs to treat both asthma and comorbidities, but when asthma was better managed over time, it caused fewer life disruptions and affected comorbidities to a lesser degree [38].

Our findings also convey important policy implications. Our cost estimates directly measure the true magnitude of asthma burden from a multimorbidity perspective, and can therefore inform policies and best practice guidelines with regard to "holistic approaches" to managing patients with asthma by also considering comorbidities. These estimates can be applied to the economic evaluation of asthma prevention and management strategies, ranging from preventive strategies to targeted asthma treatments to broad-scope interventions that aim to improve overall health or specific comorbidities in asthmatic patients.

In interpreting the findings, the limitations of this study need to be considered. First, the estimation of attributable costs could be associated with misclassification bias, because diagnostic codes can be assigned incorrectly and occasionally the diagnosis itself can be erroneous [39]. Mapping prescription drugs to disease categories is also an inexact practice [39]. Missing diagnostic information has prevented us from attributing some healthcare use records to a specific disease area. Second, our asthma case definition required a pattern of asthma-related resource use within a 12-month period [40]. This algorithm could have excluded patients with mild asthma with intermittent resource use, resulting in an upward bias in the cost estimates. Third, we reported both the costs and prevalence of comorbidity categories, but to estimate 

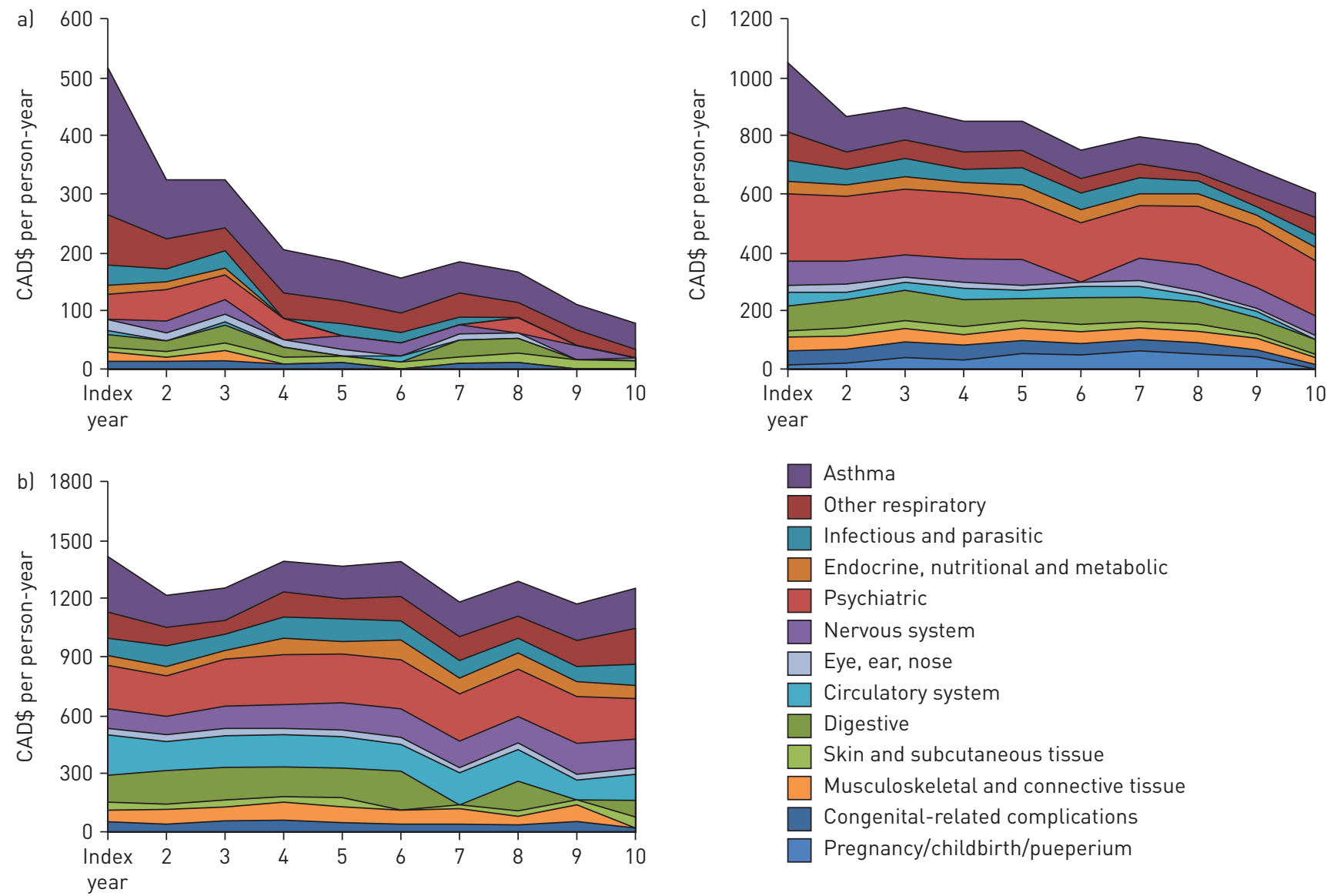

FIGURE 3 Adjusted annual excess costs by attribution to asthma and comorbidities (which cost $>\$ 10 /$ person-year) in the 10-year follow-up period from asthma onset. a) Between 5 and 18 years of age; b) between 19 and 45 years of age; c) over 45 years of age.

accurate prevalence for specific comorbid conditions requires the application of a validated condition-specific case definition, which was beyond the scope of our study.

In summary, the comorbidity-attributable excess costs in asthmatic patients were five-times higher than costs attributable to asthma, which greatly increased with age. Asthmatic patients experienced higher economic burden in many disease categories, but psychological disorders in particular had the greatest economic impact. In the world of constrained budgets, efficient resource allocation requires precise estimates of disease costs. These findings can both inform policy and clinical practice and provide insight into the disease mechanisms in asthma.

\section{Acknowledgements}

W. Chen had full access to all of the data in the study and takes responsibility for the content of the manuscript, including the integrity of the data and the accuracy of the data analysis. W. Chen, M. Sadatsafavi and T. To conceived and designed the study. L. Lynd, C.A. Marra and J.M. FitzGerald were involved in the data acquisition. W. Chen, M. Sadatsafavi, R. Balshaw and H. Tavakoli analysed the data. W. Chen wrote the first draft of the manuscript. L. Lynd, J.M. FitzGerald, C.A. Marra, R. Balshaw, T. To and H. Tavakoli contributed to the interpretation of the data and clinical inputs. All authors were involved in the revision of the manuscript for important intellectual content and approved the final version to be published.

\section{References}

1 Braman SS. The global burden of asthma. Chest 2006; 130: 4S-12S.

2 Ismaila AS, Sayani AP, Marin M, et al. Clinical, economic, and humanistic burden of asthma in Canada: a systematic review. BMC Pulm Med 2013; 13: 70.

3 Bahadori K, Doyle-Waters MM, Marra C, et al. Economic burden of asthma: a systematic review. BMC Pulm Med 2009; 9: 24.

4 Barnett SBL, Nurmagambetov TA. Costs of asthma in the United States: 2002-2007. J Allergy Clin Immunol 2011; 127: $145-152$.

$5 \quad$ Canadian Lung Association. Lung Facts 1994. Update. www.lung.ca.

6 Sadatsafavi M, Lynd L, Marra C, et al. Direct health care costs associated with asthma in British Columbia. Can Respir J 2010; 17: 74-80. 
Zhang T, Carleton BC, Prosser RJ, et al. The added burden of comorbidity in patients with asthma. J Asthma 2009; 46: 1021-1026.

8 Gershon AS, Guan J, Wang C, et al. Describing and quantifying asthma comorbidity [corrected]: a population study. PloS One 2012; 7: e34967.

9 Pérez De Llano LA, González FC, Añón OC, et al. Proyecto Camaron (Control del Asma Mediante el Análisis Regular del Óxido Nítrico). [Relationship between comorbidity and asthma control]. Arch Bronconeumol 2010; 46: 508-513.

10 Gershon AS, Wang C, Guan J, et al. Burden of comorbidity in individuals with asthma. Thorax 2010; 65: 612-618.

11 Chen W, Lynd LD, FitzGerald JM, et al. The added effect of comorbidity on health-related quality of life in patients with asthma. Qual Life Res 2015; 24: 2507-2517.

12 Xuan J, Kirchdoerfer LJ, Boyer JG, et al. Effects of comorbidity on health-related quality-of-life scores: an analysis of clinical trial data. Clin Ther 1999; 21: 383-403.

13 Government of Canada: Statistics Canada. Population by sex and age group, by province and territory (Number, both sexes). www.statcan.gc.ca/tables-tableaux/sum-som/101/cst01/demo31a-eng.htm. Date last accessed: May 23, 2016.

14 British Columbia Ministry of Health [creator]. Consolidation File (MSP Registration \& Premium Billing). Population Data BC [publisher]. Data Extract. MOH. 2014. www.popdata.bc.ca/data. Date last accessed: August 8, 2016.

15 BC Vital Statistics Agency [creator]. Vital Statistics Deaths. Population Data BC. [publisher]. Data Extract. BC Vital Statistics Agency. 2014. www.popdata.bc.ca/data. Date last accessed: August 8, 2016.

16 British Columbia Ministry of Health [creator]. Discharge Abstract Database (Hospital Separations). Population Data BC [publisher]. Data Extract. MOH. 2014. www.popdata.bc.ca/data Date last accessed: August 8, 2016.

17 British Columbia Ministry of Health [creator]. Medical Services Plan (MSP) Payment Information File. Population Data BC [publisher]. Data Extract. MOH. 2014. www.popdata.bc.ca/data. Date last accessed: August 8, 2016.

18 British Columbia Ministry of Health [creator]. PharmaNet. BC Ministry of Health [publisher]. Data Extract. Data Stewardship Committee. 2013. www.popdata.bc.ca/data. Date last accessed: August 8, 2016.

19 Prosser RJ, Carleton BC, Smith MA. Identifying persons with treated asthma using administrative data via latent class modelling. Health Serv Res 2008; 43: 733-754.

20 Hersh CP, Jacobson FL, Gill R, et al. Computed tomography phenotypes in severe, early-onset chronic obstructive pulmonary disease. COPD 2007; 4: 331-337.

21 Charlson ME, Pompei P, Ales KL, et al. A new method of classifying prognostic comorbidity in longitudinal studies: development and validation. J Chronic Dis 1987; 40: 373-383.

22 Dehejia RH, Wahba S. Propensity score matching methods for non-experimental causal studies. Rev Econ Stat 2002; 84: 151-161.

23 Government of Canada, Statistics Canada. Consumer Price Index, by province (monthly) (Canada). www.statcan. gc.ca/tables-tableaux/sum-som/101/cst01/cpis01a-eng.htm. Date last accessed: Jul 10, 2016.

24 Poole B, Robinson S, MacKinnon M. Resource intensity weights and Canadian hospital costs: some preliminary data. Healthc Manage Forum 1998; 11: 22-26.

25 McKendry R, Reid RJ, McGrail KM, et al. Emergency rooms in British Columbia: a pilot project to validate current data and describe users. Vancouver, Centre for Health Services and Policy Research, 2002.

26 ICD-10 Version:2016. apps.who.int/classifications/icd10/browse/2016/en. Date last accessed: August 8, 2016.

27 Centers for Medicare \& Medicaid Services. ICD-10-MS-DRG-Conversion-Project. 2015. www.cms.gov/Medicare/ Coding/ICD10/ICD-10-MS-DRG-Conversion-Project.html. Date last accessed: August 8, 2016.

28 The National Bureau of Economic Research. Centers for Medicare \& Medicaid Services ICD-9-CM to and from ICD-10-CM and ICD-10-PCS Crosswalk or General Equivalence Mappings. www.nber.org/data/icd9-icd-10-cmand-pcs-crosswalk-general-equivalence-mapping.html. Date last accessed: Aug 8, 2016.

29 McEvoy GK. AHFS Drug Information: Essentials 2006-2007. Bethesda, American Society of Health-System Pharmacists, 2006.

30 Cohen J. Chapter 2 - The t test for means. In: Cohen J, ed. Statistical Power Analysis for the Behavioural Sciences, revised edition. Academic Press, 1977; p. 19-74. Retrieved from www.sciencedirect.com/science/article/pii/ B9780121790608500074. Date last accessed: August 8, 2016.

31 Mihaylova B, Briggs A, O'Hagan A, et al. Review of statistical methods for analysing healthcare resources and costs. Health Econ 2011; 20: 897-916.

32 Betensky RA, Talcott JA, Weeks JC. Binary data with two, non-nested sources of clustering: an analysis of physician recommendations for early prostate cancer treatment. Biostatistics 2000; 1: 219-230.

33 Bedouch P, Marra CA, FitzGerald JM, et al. Trends in asthma-related direct medical costs from 2002 to 2007 in British Columbia, Canada: a population based-cohort study. PloS One 2012; 7: e50949.

34 Wang LY, Zhong Y, Wheeler L. Direct and indirect costs of asthma in school-age children. Prev Chronic Dis 2005; 2: A11.

35 Chen W, Thomas J, Sadatsafavi M, et al. Risk of cardiovascular comorbidity in patients with chronic obstructive pulmonary disease: a systematic review and meta-analysis. Lancet Respir Med 2015; 3: 631-639.

36 Opolski M, Wilson I. Asthma and depression: a pragmatic review of the literature and recommendations for future research. Clin Pract Epidemiol Ment Health 2005; 1: 18.

37 Patten SB, Lavorato DH. Medication use and major depressive syndrome in a community population. Compr Psychiatry 2001; 42: 124-131.

38 Ernst P, Cai B, Blais L, et al. The early course of newly diagnosed asthma. Am J Med 2002; 112: 44-48

39 Schneeweiss S, Maclure M. Use of comorbidity scores for control of confounding in studies using administrative databases. Int J Epidemiol 2000; 29: 891-898.

40 Bedouch P, Marra CA, FitzGerald JM, et al. Trends in asthma-related direct medical costs from 2002 to 2007 in British Columbia, Canada: a population based-cohort study. PloS One 2012; 7: e50949. 